\title{
Theorem on the proportionality of inertial and gravitational masses in classical mechanics
}

\author{
Andrew E. Chubykalo and Stoyan J. Vlaev \\ Escuela de Física, Universidad Autónoma de Zacatecas \\ Apartado Postal C-580 Zacatecas 98068, ZAC., México
}

(July 28, 2018)

\begin{abstract}
We considered the problem of the proportionality of inertial and gravitational masses in classical mechanics. We found that the kinetic energy of a material mass point $m$ in a circular motion with a constant angular velocity around another material point $M$ depends only on its gravitational mass. This fact, together with the known result that the straight line is a circumference with an infinite radius, allowed us to prove the proportionality between the inertial and gravitational masses.
\end{abstract}

PACS numbers: 04.20.-q, 01.55.+b 


\section{INTRODUCTION}

The equivalence principle (proportionality of the inertial $m_{i}$ and gravitational $m_{g}$ masses of a body $m$ ) is a postulate in classical mechanics. It was proven experimentally many times with a high precision. As a matter of fact, measurements verified that $m_{i}$ and $m_{g}$ may differ only by a dimensionless factor constant $\eta$

$$
m_{i}=\eta m_{g}
$$

Despite the indisputible experimental proof of this principle, a theoretical possibility of difference between inertial and gravitational masses is important for the theory (see e.g. [1-4]). That is why a proof of the equivalence principle, at least in the framework of Newtonian classical mechanics, is of great interest.

In this report we present the proof.

\section{THEOREM OF EQUIVALENCY}

\section{A. Kinetic energy of two bodies in a circular motion with a constant angular velocity}

In this paragraph we discuss a well known example from classical mechanics which showed us a way to prove the theorem. Let us consider two bodies! $m$ and $M$ with inertial and gravitational masses $m_{i}, M_{i}$ and $m_{g}, M_{g}$, respectively. The conditions for their circular motion around the center of inertia $(C I)$ with constant linear velocities $V_{m}$ and $V_{M}$ are:

$$
\frac{m_{i} V_{m}^{2}}{R-x}=G \frac{m_{g} M_{g}}{R^{2}}, \quad \frac{M_{i} V_{M}^{2}}{x}=G \frac{m_{g} M_{g}}{R^{2}}
$$

In Eq.(2) $R$ is the distance between the bodies, $x$ is the distance between the body $M$ and the $C I,(R-x)$ is the distance from the $C I$ to the body $m$ and $G$ is the gravitational constant. Eq.(2) expresses the equilibrium of the gravitational and inertial forces for both bodies. The angular velocities $\omega_{m}$ and $\omega_{M}$ are obviously equal, because the $C I$ always lies on the straight line passing through the bodies $m$ and $M$.

\footnotetext{
${ }^{1}$ In the present work we shall consider the bodies as material mass points.
} 


$$
\frac{V_{m}}{R-x}=\frac{V_{M}}{x}
$$

The system kinetic energy $K$ is:

$$
K=\frac{m_{i} V_{m}^{2}}{2}+\frac{M_{i} V_{M}^{2}}{2}
$$

If we find the velocities $V_{m}$ and $V_{M}$ from Eqs.(2,3) and substitute in Eq.(4) we obtain:

$$
K=G \frac{m_{g} M_{g}}{2 R}
$$

The fact that the inertial masses $m_{i}$ and $M_{i}$ do not enter in Eq.(5) is quite surprising. The only thing we did was to calculate the kinetic energy without postulating the equivalency of inertial and gravitational masses. The fact that the kinetic energy does not depend on the inertial masses prompts us to consider that a theoretical proof of the equivalence principle is perhaps possible in classical mechanics [5].

We found that the expression (5) is also valid in a coordinate system fixed at the body $M$.

\section{B. The proof of the Theorem}

Theorem: The inertial mass $m_{i}$ of a body $m$ is proportional to its gravitational mass $m_{g}$.

Proof: $\diamond$ We have to prove the relation (1) for an arbitrary body $m$ and for an arbitrary velocity $V$. It means we have to show that the constant $\eta$ does not depend on the masses $m_{i}, m_{g}$ and on the velocity $V$ of the body $m$.

First, we shall show that Eq.(1) is true for a given body $m$ with a given velocity $V$.

So, let some body $m$ (with inertial mass $m_{i}$ ) move along a straight line with constant velocity $V$. Its kinetic energy is:

$$
K=\frac{m_{i} V^{2}}{2}
$$

\footnotetext{
${ }^{2}$ For more details see Appendix.
} 
From a kinematic point of view, movement with constant velocity along a straight line and movement with constant (by magnitude) linear velocity along a circumference of infinite radius are equivalent.

We can also consider the movement of a similar body (with the same inertial mass $m_{i}$ ) as circumference movement around the other body $M$. The coordinate system is fixed at the body $M$. Now we can set that the kinetic energy and linear velocity of body $m$ be equal to that of case (6). Nothing can prevent us from doing it. From the condition of the equality of forces we have (see Appendix):

$$
\frac{m_{i} V^{2}}{R}=G \frac{m_{g} M_{g}}{R^{2}}
$$

where $m_{g}, M_{g}$ are gravitational masses of bodies $m$ and $M, R$ is the distance between $m$ and $M$. Expressing " $V$ " from (7) and substituting it into the formula of the kinetic energy of the body $m$ we obtain (see Eq.(5)):

$$
K=G \frac{m_{g} M_{g}}{2 R}
$$

Let us now increase $M_{g}$ and $R$ conserving at the same time the value of $K$. In other words, we consider a series of independent circular motions: in each of them the same body $m$ has the same velocity $V$ (and, of course, the same kinetic energy $K$ ) and besides, the values of $M_{g}$ and $R$ increase appropriately. In this case $M_{g}(R)$ and $R\left(M_{g}\right)$ are oneto-one functions. It is obvious (see (8)) that if $K, m_{g}$ and $G$ are constants, $M_{g}(R)$ and $R$ will be linear dependent (proportional) functions, i.e.

$$
M_{g}(R)=C \cdot R,
$$

where $C$ is some suitable dimensional constant (for the considered $m_{i}, m_{g}$ and $V$, of course). After substituting the last formula in (8) and tending $R$ to infinity (conserving

\footnotetext{
${ }^{3}$ Note that if $R$ tends to infinity, both forces (gravitational $F_{g}=G m_{g} M_{g} / R^{2}$ and inertial $\left.F_{i}=m_{i} V^{2} / R\right)$ tend to zero:

$$
F_{g}=G \frac{m_{g}}{R}\left(\frac{M_{g}}{R}\right)=G \frac{m_{g} C}{R} \rightarrow 0, \quad F_{i}=\frac{m_{i} V^{2}}{R} \rightarrow 0,
$$

where $m_{i}, m_{g}, C$ and $V$ have finite constant values. A constant velocity $V$ when $R$ tends to infinity means that the angular velocity $\omega=V / R$ tends to zero. So we reach a situation without any forces when the body $m$ moves with a constant velocity $V$.
} 
at the same time values of $K, m_{g}$ and $G$ ) we obtain from (8)

$$
K=m_{g} \frac{G C}{2}
$$

where "GC" has dimension of " $V$ ". It means that (9) can be rewritten as

$$
K=m_{g} \eta \frac{V^{2}}{2}
$$

here $\eta$ is a dimensionless non-defined constant.

Recalling the above-mentioned remark (equivalence between straight line movement and the movement along the circumference of the infinite radius), we conclude that the kinetic energy of the body moving along the straight line with constant velocity is proportional to the gravitational mass. Comparing (10) and (6) we obtain the equality

$$
m_{i}=\eta m_{g}
$$

where $\eta$ is a constant non-defined in the framework of the above-mentioned considerations.

Now we have to prove the relation (1) (or (11)) for an arbitrary body $m$ and for an arbitrary velocity $V$. It means we have to show that the constant $\eta$ does not depend on the masses $m_{i}, m_{g}$ and on the velocity $V$ of the body $m$.

We shall now show that $\eta$ does not depend on the velocity $V$, which the same body can have if it moves around $M$ but at a different distance $R$. If we apply the same argument separately for the velocities $V_{1}$ and $V_{2}$ of the same body $m$ we will find:

$$
\begin{aligned}
& K_{1}=\frac{m_{i} V_{1}^{2}}{2}=G \frac{m_{g} M_{g}}{2 R_{1}}=G \frac{m_{g} C_{1}}{2}=\frac{m_{g} \eta_{1} V_{1}^{2}}{2} \\
& K_{2}=\frac{m_{i} V_{2}^{2}}{2}=G \frac{m_{g} M_{g}}{2 R_{2}}=G \frac{m_{g} C_{2}}{2}=\frac{m_{g} \eta_{2} V_{2}^{2}}{2}
\end{aligned}
$$

In the expressions (12) $\eta_{1}=G C_{1} / V_{1}^{2}$ and $\eta_{2}=G C_{2} / V_{2}^{2}$ (see Eqs. $(9,10)$ ). The constants $C_{1}$ and $C_{2}$ have a meaning as in (9) but for the distances $R_{1}$ and $R_{2}$ respectively. Now from (12) follows:

$$
m_{i}=\eta_{1} m_{g} \quad m_{i}=\eta_{2} m_{g}
$$


and consequently

$$
\eta_{1}=\eta_{2}=\eta
$$

Eq.(14) means that the constant $\eta$ does not depend on the velocity.

The last point is to prove that Eq.(1) is true for arbitrary masses $m_{i}$ and $m_{g}$. We know that (1) is true for a body $m$ with masses $m_{i}$ and $m_{g}$. Let us now consider another body $\widetilde{m}$ with masses $\widetilde{m}_{i}$ and $\widetilde{m}_{g}$ proportional with a different constant $\widetilde{\eta}$.

$$
\widetilde{m}_{i}=\widetilde{\eta} \widetilde{m}_{g}
$$

Additivity of inertial masses gives:

$$
m_{i}+m_{i}=2 m_{i} \equiv \mathcal{M}_{i}
$$

In its turn, additivity of gravitational masses gives too:

$$
m_{g}+m_{g}=2 m_{g} \equiv \mathcal{M}_{g}
$$

But we know that $m_{i}=\eta m_{g}$, so

$$
\mathcal{M}_{i}=m_{i}+m_{i}=\eta m_{g}+\eta m_{g}=\eta\left(m_{g}+m_{g}\right)=\eta 2 m_{g}=\eta \mathcal{M}_{g}
$$

Eq.(18) shows that the masses $\mathcal{M}_{i}$ and $\mathcal{M}_{g}$ are proportional with the same constant $\eta$. Obviously in this particular case the new masses $\mathcal{M}_{i}$ and $\mathcal{M}_{g}$ are different from the masses $m_{i}$ and $m_{g}$. It is clear that Eqs.(15-18) can be generalized:

$$
\widetilde{m}_{i}=b m_{i} \quad \widetilde{m}_{g}=b m_{g}
$$

where $b$ is an arbitrary real number. We also have :

$$
\widetilde{m}_{i}=b m_{i}=b \eta m_{g}=\eta b m_{g}=\eta \widetilde{m}_{g}
$$

From Eqs.(15) and (20) follows:

$$
\eta=\widetilde{\eta}
$$

The constant $\eta$ does not depend on the masses. Now the proof is finished. $\diamond$ 


\section{CONCLUSION}

The proof of the theorem of the equivalency is based on three facts . The first is the well-known result of the Euclidean geometry that a circumference with an infinite radius $R$ coincides with a straight line. The second is that the kinetic energy of a body $m$ in a straight linear motion with a constant velocity $V$ is equal to the kinetic energy of this body in a circular motion with a linear velocity $V$ around the body $M$. The third fact is that the kinetic energy of two bodies in a circular motion was expressed only with their gravitational masses.

In this way we proved that the inertial and gravitational masses are proportional.

\section{Appendix}

We have introduced two rectangular coordinate systems $\mathcal{K}$ and $\tilde{\mathcal{K}}$. The inertial system $\mathcal{K}$ is connected with the center of inertia $(C I)$ of the bodies (material mass points). The non-inertial coordinate system $\widetilde{\mathcal{K}}$ has its origin $\widetilde{\mathcal{O}}$ at the body $M$ and consequently moves with respect to the system $\mathcal{K}$ with a linear velocity $\mathbf{V}_{M}$ (its magnitude $V_{M}$ is constant) around the point $\mathcal{O}(C I)$ along a circular trajectory. We have chosen the planes $\widetilde{\mathcal{X O Y}}$ and $\mathcal{X O Y}$ to coincide and consequently the axes $\widetilde{\mathcal{O Z}}$ and $\mathcal{O Z}$ are parallel. The system $\widetilde{\mathcal{K}}$ also rotates around axis of rotation $\widetilde{\mathcal{O Z}}$ with an non-zero angular velocity $\boldsymbol{\omega}=(0,0, \omega)$. An observer in $\widetilde{\mathcal{K}}$ will see that the body $m$ moves in the plane $\widetilde{\mathcal{X O} Y}$ along a circumference of radius $R$ around the body $M$.

The equation of motion in the system $\widetilde{\mathcal{K}}$ is:

$$
m_{i} \mathbf{a}_{(\mathrm{rel})}=\mathbf{F}_{g}+\mathbf{F}_{i n}
$$

where

$$
\mathbf{F}_{\text {in }}=2 m_{i}\left(\mathbf{V}_{(\mathrm{rel})} \times \boldsymbol{\omega}\right)-m_{i} \dot{\mathbf{V}}_{M}+m_{i} \omega^{2} \mathbf{R}-m_{i}(\dot{\boldsymbol{\omega}} \times \mathbf{R})
$$

In Eq.(22) $\mathbf{a}_{(\mathrm{rel})}$ and $\mathbf{V}_{(\mathrm{rel})}$ are respectively the acceleration and velocity of the relative motion of the body $m$ with inertial mass $m_{i}$ in the system $\widetilde{\mathcal{K}}, \mathbf{R}$ is the radius-vector of the material point $m$ in $\widetilde{\mathcal{K}}$ with components $\left(x_{(\mathrm{rel})}, y_{(\mathrm{rel})}, 0\right), \mathbf{F}_{g}$ is the gravitational force.

For $\omega=$ const., we have:

$$
a_{(\mathrm{rel})}=\frac{V_{(\mathrm{rel})^{2}}}{R}
$$


Let see now, for $\omega=$ const., may we have

$$
\mathbf{F}_{\text {in }}=\mathbf{0}
$$

which could allow us to write the equation of motion (7) in $\widetilde{\mathcal{K}}$ ? The eq.(24) gives two equalities:

$$
\left\{\begin{array}{l}
2 m_{i} \omega V_{(\mathrm{rel}) y}-m_{i} \dot{V}_{M x}+m_{i} \omega^{2} x_{(\mathrm{rel})}=0 \\
2 m_{i} \omega V_{(\mathrm{rel}) x}-m_{i} \dot{V}_{M y}+m_{i} \omega^{2} y_{(\mathrm{rel})}=0
\end{array}\right.
$$

After elimination of $\omega^{2}$ we find:

$$
2 \omega\left(x_{(\mathrm{rel})} V_{(\mathrm{rel}) x}+y_{(\mathrm{rel})} V_{(\mathrm{rel}) y}\right)=x_{(\mathrm{rel})} \dot{V}_{M y}-y_{(\mathrm{rel})} \dot{V}_{M x}
$$

The left part of (26) is zero, because

$$
x_{(\mathrm{rel})}^{2}+y_{(\mathrm{rel})}^{2}=R^{2}(=\text { const. })
$$

and the time differentiation of (27) gives:

$$
x_{(\mathrm{rel})} \dot{x}_{(\mathrm{rel})}+y_{(\mathrm{rel})} \dot{y}_{(\mathrm{rel})}=0=x_{(\mathrm{rel})} V_{(\mathrm{rel}) x}+y_{(\mathrm{rel})} V_{(\mathrm{rel}) y}
$$

Consequently, for $\omega=$ const, the eq.(24) fulfills if the right side of eq.(26) is zero, which means that the next equality should be true:

$$
\left\{\begin{array}{c}
x_{(\mathrm{rel})} \dot{V}_{M y}=y_{(\mathrm{rel})} \dot{V}_{M x} \\
x_{(\mathrm{rel})} / y_{(\mathrm{rel})}=\dot{V}_{M x} / \dot{V}_{M y}
\end{array}\right.
$$

To verify if (29) is true we shall make some considerations. First, let define a vector $\mathbf{V}_{(\mathrm{tr})}$ :

$$
\mathbf{V}_{(\operatorname{tr})}=\mathbf{V}_{M}+\boldsymbol{\omega} \times \mathbf{R}
$$

The vectors $\mathbf{V}_{(\operatorname{tr})}$ and $\mathbf{V}_{M}$ are collinear because the vectors $\boldsymbol{\omega}$ and $\mathbf{V}_{M}$ are both perpendicular to the vector $\mathbf{R}$.

$$
\mathbf{V}_{(\operatorname{tr})}=k \mathbf{V}_{M}
$$

where $k$ is a scalar factor. From (30) and (31) we have: 


$$
\left\{\begin{array}{l}
k V_{M x}=V_{M x}-\omega y_{(\mathrm{rel})} \\
k V_{M y}=V_{M y}+\omega x_{(\mathrm{rel})}
\end{array}\right.
$$

If we eliminate $\omega$ in (32), will find:

$$
\left\{\begin{array}{c}
x_{(\mathrm{rel})} V_{M x}=y_{(\mathrm{rel})} V_{M y} \\
x_{(\mathrm{rel})} / y_{(\mathrm{rel})}=-V_{M y} / V_{M x}
\end{array}\right.
$$

The body $M$ moves around $C I$ with a linear velocity $\mathbf{V}_{M}$ which has a constant magnitude $V_{M}$.

$$
V_{M x}^{2}+V_{M y}^{2}=V_{M}^{2}(=\text { const. })
$$

After time differentiation of (34) we obtain:

$$
\left\{\begin{array}{l}
V_{M x} \dot{V}_{M x}+V_{M y} \dot{V}_{M y}=0 \\
\dot{V}_{M x} / \dot{V}_{M y}=-V_{M y} / V_{M x}
\end{array}\right.
$$

Then from (33) and (35) follows:

$$
x_{(\mathrm{rel})} / y_{(\mathrm{rel})}=\dot{V}_{M x} / \dot{V}_{M y}
$$

So we have found that the relation (36) is true. But eq.(36) is the second equation in (29). So the eq.(29) and consequently eq.(24) fulfill. Thus, we have shown that it is possible to choose $\omega=$ const., for which the equation of motion in the system $\widetilde{\mathcal{K}}$ looks like:

$$
m_{i} \mathbf{a}_{(\mathrm{rel})}=\mathbf{F}_{g}
$$

Now from (23) and (37) we find:

$$
\frac{m_{i} V_{(\mathrm{rel})}^{2}}{R}=G \frac{m_{g} M_{g}}{R^{2}}
$$

Obviously eq.(38) is the same as eq.(7). Note that the velocity $V_{(\mathrm{rel})}$ in Appendix coincides with the velocity $V$ in SECTION II.

\section{Acknowledgments}

We are grateful to Dr. Dharam V. Ahluvalia and Prof. Valeri V. Dvoeglazov for many stimulating discussions and critical comments. 


\section{REFERENCES}

[1] E. Mach, Mechanics (St. Petersburg, 1906).

[2] M. Sachs, Brit. J. Phil. Sci. 27, 225 (1976).

[3] P. Graneau, The riddle of inertia (Electronic World and World 96, 1990).

[4] A. Assis, Found. Phys. Lett. 2, 301 (1989).

[5] A. Chubykalo, Hadronic Journal 19, 610 (1996). 